\title{
EVALUATION OF THE ANTIFUNGAL ACTIVITY BY PLANT EXTRACTS AGAINST Colletotrichum gloeosporioides PENZ
}

\author{
Avaliação da atividade anti-fúngica de extratos vegetais contra \\ Colletotrichum gloeosporioides Penz
}

\author{
Polyanna Alves Silva ${ }^{1}$, Denilson Ferreira Oliveira ${ }^{2}$, Ney Robson Taironi do Prado ${ }^{3}$, \\ Douglas Antônio de Carvalho ${ }^{4}$, Gilvane Aparecida de Carvalho ${ }^{5}$
}

\begin{abstract}
Aiming to develop more efficient and environmental friendly methods than those available to control Colletotrichum gloeosporioides Penz, which causes blister spot in coffee trees, a search for plants able to produce substances active against such pathogen was carried out. Thus, extracts of 48 plant species, collected at Alto Rio Grande region, in Minas Gerais, were prepared and submitted to in vitro assays with that fungus. The best results were obtained with the extracts prepared from Digitalis lanata Ehrh, Origanum manjorona L., Plantago lanceolata Hook. and Stevia rebaudiana (Bertoni) Bertoni, which inhibited C. gloeosporioides spores germination. After dilution of some active extracts with aqueous $1 \%$ Tween 80 solution in a 1:2 or 1:3 ratio (extract:aqueous solution), their antifungal activity vanished. Some of the active extracts were also submitted to freeze drying and none of them presented any alteration in their antifungal activity. Concluding, several plants presented potential to be used in the search for new bioactive substances to control $C$. gloeosporioides, especially $O$. manjorona L., which inhibited $96 \%$ of the fungus spores germination.
\end{abstract}

Index terms: Disease control, biocide, Coffee's blister spot.

\section{RESUMO}

Com vistas a contribuir para o desenvolvimento de métodos mais eficientes e menos agressivos ao ambiente que aqueles disponíveis para o controle de Colletotrichum gloeosporioides Penz, causador da mancha manteigosa em cafeeiros, buscou-se identificar espécies vegetais produtoras de substâncias ativas contra o referido patógeno. Para tanto, prepararam-se extratos de 48 espécies vegetais, coletadas na região do Alto Rio Grande, em Minas Gerais, para serem submetidos a testes in vitro com o mencionado fungo. Constatou-se que os melhores resultados foram obtidos com os extratos oriundos de Digitalis lanata Ehrh, Origanum manjorona L., Plantago lanceolata Hook. e Stevia rebaudiana (Bertoni) Bertoni que inibiram a germinação dos esporos de $C$. gloeosporioides. Alguns dos extratos ativos também foram submetidos a diluições em solução aquosa de Tween 80 a $1 \%$, o que permitiu observar que, na proporção de 1:2 ou 1:3 (extrato: solução aquosa), os extratos perdiam a atividade antifúngica. Verificouse também que alguns dos extratos ativos não alteraram suas atividades quando submetidos ao processo de liofilização. Desse modo, identificaram-se várias plantas promissoras na pesquisa por novas substâncias bioativas para o controle de C. gloeosporioides, com destaque para $O$. manjorona L., que inibiu $96 \%$ da germinação dos esporos do referido fungo.

Termos para indexação: Controle de doenças, biocida, mancha manteigosa do cafeeiro.

\section{(Received in august 4, 2006 and approved in march 15, 2007)}

\section{INTRODUCTION}

Coffee is one of the most important export crops for Brazil, which is the leading producer and exporter of this commodity. With an area of 2.2 million ha devoted to coffee plants, such country produced 28.82 million sacks of coffee during the year of 2004 and exported the equivalent to US\$ 1.51 billions in coffee products at the same year (BRASIL, 2005).
Among the phytopathological problems that occur during the Coffea arabica L. cultivation are those caused by fungi like Colletotrichum spp., whose presence in several coffee producing Brazilian regions has been constantly reported (CARVALHO, 2004). The symptoms of the fungal parasitism vary in accordance with the species, resulting in different names for the corresponding diseases. One of the most important is the blister spot in coffee plants, which is caused by the

\footnotetext{
${ }^{1}$ Química, Doutoranda - Departamento de Química/DQI - Universidade Federal de Lavras/UFLA - Cx. P. 3037-37200-000 - Lavras, MG - polyanna@ufla.br ${ }^{2}$ Químico, Doutor, Professor Associado - Departamento de Química/DQI - Universidade Federal de Lavras/UFLA - Cx. P. 3037 - $37200-000$ - Lavras, MG denilson@ufla.br

${ }^{3}$ Acadêmico do curso de Química - Departamento de Química/DQI - Universidade Federal de Lavras/UFLA - Cx. P. 3037 - $37200-000$ - Lavras, MG neyquimica@gmail.com

${ }^{4}$ Engenheiro agrônomo, Doutor, Professor Titular - Departamento de Biologia/DBI - Universidade Federal de Lavras/UFLA - Cx. P. 3037 - $37200-000$ Lavras, MG - douglasc@ufla.br

${ }^{5}$ Engenheira agrônoma, Mestre - Departamento de Fitopatologia/DFP - Universidade Federal de Lavras/UFLA - Cx. P. 3037 - $37200-000$ - Lavras, MG gac@yahoo.com.br
}

Ciênc. agrotec., Lavras, v. 32, n. 2, p. 420-428, mar./abr., 2008 
fungus Colletotrichum gloeosporioides Penz (MIRANDA, 2003).

Traditionally, plant fungal diseases are controlled by synthetic fungicides, which increase agricultural costs and contaminate the environment with very toxic substances (CARVALHO, 2004). A possible alternative to solve such problem is the use of plants able to produce antifungal substances (MIRANDA, 2003). Among the several examples available in the literature are Zanthoxylum americanum Mill. and Piper regnellii var. pallescens (C. DC.) Yunck, whose antifungal activities are well known (BAFI-YEBOA et al., 2005; PESSINI et al., 2005).

Thus, this work aimed to contribute for the developement of new methods to control fungi in coffee plants by the identification of plant extracts active against C. gloeosporioides.

\section{MATERIAL AND METHODS}

Fresh leaves $(3.0 \mathrm{~g})$ of several plants (Table 1) collected in the Alto Rio Grande Region, State of Minas Gerais, Brazil, were cut in small pieces and soaked in methanol during $48 \mathrm{~h}$. The resulting mixtures were filtered and the insoluble parts were soaked in more methanol during the same period of time. The new mixtures were filtered and the liquids obtained during both filtrations were combined and concentrated to dryness in a rotary evaporator. Immediately before carrying out the tests, the dry residues were dissolved in an aqueous $1 \%(\mathrm{~g} / \mathrm{mL})$ Tween 80 solution $(30 \mathrm{~mL})$, resulting in the extracts to be studied.

C. gloeosporioides, isolated by Miranda (2003) and kept on deposit at Departamento de Fitopatologia Universidade Federal de Lavras, was transferred to Petri dishes containing the culture medium MEA (20 g Agar, 20 $\mathrm{g}$ malt extract and $1 \mathrm{~L}$ distilled water), which was previously autoclaved at $120^{\circ} \mathrm{C}$. After seven days at $22^{\circ} \mathrm{C}$, under $12 \mathrm{~h}$ photoperiods, sterilized distilled water was poured into the dishes and, with a sterilized small paintbrush, the spores were removed. They were counted in the resulting suspension by the use of a Neubauer chamber and water was added to reach $4.0 \times 10^{4}$ spores $/ \mathrm{mL}$.

As described by Carvalho (2004), the spore suspension $(40 \mu \mathrm{L})$ was poured into each well of three well glass slides, which were kept inside Petri dishes. The plant extracts dissolved in aqueous Tween $80(40 \mu \mathrm{L})$ were also poured into the wells and, after seven days at $22^{\circ} \mathrm{C}$, which was the best condition for maximum spore germination, 20 $\mathrm{mL}$ of a lactoglycerol solution (20 g lactic acid, $40 \mathrm{~g}$ glycerol, $20 \mathrm{~mL}$ distilled water and $0.05 \mathrm{~g}$ trypan blue) was poured into all wells to stop the germination. The wells were divided into five identical parts, in which 20 spores were counted. The percentage of germinated spores in each well was obtained after summing the values of germinated spores in each part.

All tests were carried out with three repetitions, arranged in a randomized design, employing aqueous $1 \%$ Tween 80 solution as control. Statistical calculations were done using SISVAR software (FERREIRA, 2000) and values of germinated spores $(\%)$ underwent variance analysis using ANOVA; means were analyzed according to the Scott and Knott test $(P \leq 0.05)$. According to Banzatto \& Kronka (1989), the observed heterogeneity of variances (9:1) was above the recommended (7:1) for a joint statistical analysis. As a consequence, the experiments underwent statistical calculation separated from each other.

Some of the extracts affording low values of germinated spores were tested again at different concentrations: 1:0, 1:1 and 1:3 (plant extract dissolved in 1\% Tween 80: $1 \%$ Tween 80 ). Statistical analysis were done as described above. However, as the homogeneity of variances was observed, a joint statistical calculation was performed.

The most promising extracts were once more prepared from $1.0 \mathrm{~g}$ of fresh plant material. Nevertheless, after the solvent removal, half of each residue were freezedried before dissolution in aqueous Tween 80 solution. The resulting extracts were submitted to the test with $C$. gloeosporioides as described above and values of germinated spores $(\%)$ underwent a non-parametric statistical analysis, employing the Wilcoxon test for matched pairs (SIEGEL, 1977). A $P$-value $<0.05$ was accepted as statistically significant.

\section{RESULTS AND DISCUSSION}

No influence on $C$. gloeosporioides was observed when the corresponding spores were treated with the extracts of C. jobi, C. officinalis, D. pectinata, H. rosa, M. pulegium, N. catarica, N. tabacum, Origanum sp., $P$. sonchifolia and T. officinale (Table 1). This seemed a reasonable result, since no report in the literature was found about the antifungal activity of such plants.

Although the activity against fungi has already been described for the extracts of A. arborescens, $H$. perforatum, J. curcas, $P$. granatum, $P$. graveolens, $S$. officinale, S. officinalis and S. nigra (DAMME et al., 2002; GIAMPERI et al., 2002; HOLETZ et al., 2002; KAWAI et al., 1998; LU et al., 2002; SENER, 1994; SHIN, 2003; THANGAVELU et al., 2004), none of them influenced the germination of C. gloeosporioides spores (Table 1). 
Table 1 - Percentage of Colletotrichum gloeosporioides germinated spores after treatment with plant extracts at a constant concentration.

\begin{tabular}{|c|c|c|c|}
\hline Family & $\begin{array}{l}\text { Scientific } \\
\text { name }\end{array}$ & $\begin{array}{l}\text { Vernacular } \\
\text { name }\end{array}$ & $\begin{array}{l}\text { Germinated } \\
\text { spores }(\%)\end{array}$ \\
\hline \multicolumn{4}{|l|}{ Experiment 1} \\
\hline Poaceae & Coix lacryma-jobi L. & Job's tears & $62.6 \mathrm{a}$ \\
\hline Euphorbiaceae & Jatropha curcas L. & Barbados nut & $69.3 \mathrm{a}$ \\
\hline \multirow[t]{2}{*}{ Boraginaceae } & Symphytum officinale L. & Comfrey & $67.0 \mathrm{a}$ \\
\hline & $1 \%$ Tween 80 & & $68.0 \mathrm{a}$ \\
\hline \multicolumn{4}{|l|}{ Experiment 2} \\
\hline Asteraceae & Achillea millefolium $\mathrm{L}$. & Common yarrow & $58.3 \mathrm{~b}$ \\
\hline Asphodelaceae & Aloe saponaria (Aiton) Haw & African aloe & $50.3 \mathrm{a}$ \\
\hline \multirow[t]{2}{*}{ Gleicheniaceae } & Dicranopteris pectinata (Willd.) Und. & Fern & $76.0 \mathrm{c}$ \\
\hline & $1 \%$ Tween 80 & & $73.6 \mathrm{c}$ \\
\hline \multicolumn{4}{|l|}{ Experiment 3} \\
\hline Asteraceae & Achillea millefolium $\mathrm{L} . *$ & Common yarrow & $58.6 \mathrm{a}$ \\
\hline Rosaceae & Agrimonia eupatoria L. & $\begin{array}{l}\text { Common } \\
\text { agrimony }\end{array}$ & $52.6 \mathrm{a}$ \\
\hline \multirow[t]{2}{*}{ Asteraceae } & Calendula officinalis L. & English marigold & $74.0 \mathrm{a}$ \\
\hline & $1 \%$ Tween 80 & & $63.6 \mathrm{a}$ \\
\hline \multicolumn{4}{|l|}{ Experiment 4} \\
\hline Asteraceae & Arctium lappa (Hill) Bernh. & Greater burdock & $52.6 \mathrm{a}$ \\
\hline \multirow[t]{2}{*}{ Asphodelaceae } & Aloe arborescens Mill. & Krantz aloe & $75.6 \mathrm{~b}$ \\
\hline & $1 \%$ Tween 80 & & $74.0 \mathrm{~b}$ \\
\hline \multicolumn{4}{|l|}{ Experiment 5} \\
\hline Apiaceae & Foeniculum vulgare Mill. & Sweet fennel & $85.0 \mathrm{c}$ \\
\hline \multirow[t]{4}{*}{ Lamiaceae } & Glechoma hederacea L. & Ground ivy & $81.3 \mathrm{c}$ \\
\hline & Mentha pulegium $\mathrm{L}$. & Pennyroyal & $68.3 \mathrm{~b}$ \\
\hline & Nepeta cataria L. & Catnip & $72.0 \mathrm{~b}$ \\
\hline & Ocimum gratissimum $\mathrm{L}$. & African basil & $13.0 \mathrm{a}$ \\
\hline \multirow[t]{2}{*}{ Geraniaceae } & Pelargonium graveolens L'Hér & Geranium & $70.6 \mathrm{~b}$ \\
\hline & $1 \%$ Tween 80 & & $67.3 \mathrm{~b}$ \\
\hline \multicolumn{4}{|l|}{ Experiment 6} \\
\hline Lamiaceae & Mentha spicata $\mathrm{L}$. & Spearmint & $96.6 \mathrm{~d}$ \\
\hline Nyctaginaceae & Mirabilis jalapa $\mathrm{L}$. & Four o'clock flower & $95.3 \mathrm{~d}$ \\
\hline Moringaceae & Moringa oleifera Lam. & Moringa & $88.3 \mathrm{~d}$ \\
\hline Rutaceae & Ruta graveolens $\mathrm{L}$. & Rue & $06.6 \mathrm{a}$ \\
\hline \multirow[t]{2}{*}{ Lamiaceae } & Thymus vulgaris $\mathrm{L}$. & Thyme & $15.0 \mathrm{~b}$ \\
\hline & $1 \%$ Tween 80 & & $50.0 \mathrm{c}$ \\
\hline
\end{tabular}


Table 1 - Continued...

\begin{tabular}{|c|c|c|c|}
\hline Family & $\begin{array}{l}\text { Scientific } \\
\text { name }\end{array}$ & $\begin{array}{l}\text { Vernacular } \\
\text { name }\end{array}$ & $\begin{array}{l}\text { Germinated } \\
\text { spores }(\%)\end{array}$ \\
\hline \multicolumn{4}{|l|}{ Experiment 7} \\
\hline Clusiaceae & Hypericum perforatum $\mathrm{L}$. & St. John's wort & $87.3 \mathrm{a}$ \\
\hline \multirow[t]{2}{*}{ Lamiaceae } & Origanum sp. & & $76.6 \mathrm{a}$ \\
\hline & Salvia officinalis L. & Sage & $72.0 \mathrm{a}$ \\
\hline \multirow[t]{2}{*}{ Caprifoliaceae } & Sambucus nigra L. & Black elderberry & $75.0 \mathrm{a}$ \\
\hline & $1 \%$ Tween 80 & & $74.3 \mathrm{a}$ \\
\hline \multicolumn{4}{|l|}{ Experiment 8} \\
\hline Plantaginaceae & Plantago lanceolata Hook. & Narrowleaf plantain & $25.3 \mathrm{a}$ \\
\hline \multirow[t]{2}{*}{ Punicaceae } & Punica granatum $\mathrm{L}$. & Pomegranate & $72.0 \mathrm{~b}$ \\
\hline & $1 \%$ Tween 80 & & $70.3 \mathrm{~b}$ \\
\hline \multicolumn{4}{|l|}{ Experiment 9} \\
\hline Rubiaceae & Coffea arabica $\mathrm{L}$. & Coffee & $84.0 \mathrm{c}$ \\
\hline Rutaceae & Citrus sp. & & $05.3 \mathrm{a}$ \\
\hline Scrophulariaceae & Digitalis lanata Ehrh & Woolly foxglove & $18.3 \mathrm{~b}$ \\
\hline Malpighiaceae & Malpighia glabra L. & Acerola & $95.6 \mathrm{~d}$ \\
\hline Phytolacaceae & Petiveria alliacea $\mathrm{L}$. & Anamu & $08.3 \mathrm{a}$ \\
\hline Anacardiaceae & Schinus molle L. & Brazillian peppertree & $20.6 \mathrm{~b}$ \\
\hline \multirow[t]{2}{*}{ Asteraceae } & Stevia rebaudiana (Bertoni) Bertoni & Sweetleaf & $17.0 \mathrm{~b}$ \\
\hline & $1 \%$ Tween 80 & & $77.6 \mathrm{c}$ \\
\hline \multicolumn{4}{|l|}{ Experiment 10} \\
\hline Malvaceae & Hibiscus rosa sinensis $\mathrm{L}$. & Chinese hibiscus & $96.3 \mathrm{~d}$ \\
\hline \multirow[t]{2}{*}{ Lamiaceae } & Melissa officinalis L. & Lemon balm & $66.3 \mathrm{~b}$ \\
\hline & Origanum majorona L. & Oregano & $03.3 \mathrm{a}$ \\
\hline Asteraceae & Parthenium hysterophorus L. & Salvinia & $79.0 \mathrm{c}$ \\
\hline Plantaginaceae & Plantago major L. & Common plantain & $63.6 \mathrm{~b}$ \\
\hline \multirow[t]{2}{*}{ Asteraceae } & Polymnia sonchifolia Poepp. & Yacon & $90.0 \mathrm{~d}$ \\
\hline & $1 \%$ Tween 80 & & $91.3 \mathrm{~d}$ \\
\hline \multicolumn{4}{|l|}{ Experiment 11} \\
\hline Rutaceae & Citrus limon (L.) Burm. f. & Lemon & $01.6 \mathrm{a}$ \\
\hline \multirow[t]{2}{*}{ Onagraceae } & Oenothera biennis L. & $\begin{array}{l}\text { Common evening } \\
\text { primrose }\end{array}$ & $48.6 \mathrm{~b}$ \\
\hline & $1 \%$ Tween 80 & & $78.6 \mathrm{c}$ \\
\hline \multicolumn{4}{|l|}{ Experiment 12} \\
\hline Rubiaceae & $\begin{array}{l}\text { Coffea arabica L. cv. Acaiá-Cerrado } \\
\text { MG1474) }\end{array}$ & Coffee & $84.3 \mathrm{a}$ \\
\hline \multirow[t]{2}{*}{ Zingiberaceae } & Curcuma longa $\mathrm{L}$. & Turmeric & $87.3 \mathrm{a}$ \\
\hline & $1 \%$ Tween 80 & & $97.3 \mathrm{a}$ \\
\hline
\end{tabular}


Table 1 - Continued...

\begin{tabular}{llll}
\hline \multicolumn{1}{c}{ Family } & \multicolumn{1}{c}{$\begin{array}{c}\text { Scientific } \\
\text { name }\end{array}$} & \multicolumn{1}{c}{$\begin{array}{c}\text { Vernacular } \\
\text { name }\end{array}$} & $\begin{array}{c}\text { Germinated } \\
\text { spores }(\%)\end{array}$ \\
\hline $\begin{array}{l}\text { Experiment } 13 \\
\text { Zingiberaceae }\end{array}$ & Curcuma zedoaria (Christm.) Roscoe & Zedoary & $33.3 \mathrm{a}$ \\
Solanaceae & Nicotiana tabacum L. & Tobacco & $90.6 \mathrm{c}$ \\
Phytolacaceae & Petiveria sp. & Common dandelion & $55.3 \mathrm{~b}$ \\
Asteraceae & Taraxacum officinale Weber & $90.3 \mathrm{c}$ \\
& 1\% Tween 80 & $88.0 \mathrm{c}$ \\
\hline
\end{tabular}

Means of six replicates with the same letter do not differ significantly $(P \leq 0.05)$. CV values varied between 3.5 and $14.3 \%$.

*A mixture of leaves and inflorescences was used to prepare the extract.

An unexpected result was obtained for the extracts of F. vulgare, G. hederaceae, M. glabra, M. spicata and $M$. oleifera, whose antifungal activity has already been described in the literature (ABOU-JAWDAH et al., 2002; ADAM et al., 1998; CACERES et al., 1993; CORTEZ et al., 1998; LIMA et al., 1992; PATRA et al., 2002). Somehow, they increased the germination of $C$. gloeosporioides spores (Table 1).

The extracts of A. lappa, A. millefolium, Citrus sp., C. limon, C. longa, C. zedoarea, M. officinalis, O. biennis, O. gratissimum, P. alliacea, P. hysterophorus, $P$. major, $R$. graveolens and $T$. vulgaris were all active against $C$. gloeosporioides (Table 1), what is in accordance with the activity previously described against fungi from other genus (BENEVIDES et al., 2001; FICKER et al., 2003; FIORI et al., 2000; GIAMPERI et al., 2002; HOLETZ et al., 2002; KISHORE et al., 1996; MISHRA et al., 2002; NINO et al., 2003; POPOVIC et al., 2002; ROTH et al., 1998; SHRIVASTAVA et al., 1984; SHUKLA et al., 1999; SOUZA et al., 2005; TIWARI et al., 2003). Specifically for $R$. graveolens, the activity of the corresponding extract against $C$. gloesporioides was already known (OLIVA et al., 2003).

The extract of $C$. arabica cv. Acaiá-Cerrado MG1474 was also studied, since this coffee plant was less susceptible to $C$. gloeosporioides (FERREIRA, 2004) than other cultivars. Nevertheless, only a weak inhibition of spore germination was observed (Table 1).

A. eupatoria and Petiveria sp. extracts presented moderate antifungal action, while those from $D$. lanata, $P$. lanceolata and $S$. rebaudiana afforded low values of germinated spores. One of the best results was observed for the extract of $O$. manjorona, which inhibited about 96 $\%$ of the spores. These results were a surprise, since no report about the antifungal activity of such plants was found.

In order to assess the effect of extracts concentration on C. gloeosporioides spores, six of them were randomly selected among extracts presenting antifungal activity. None of them influenced spore germination in a 1:3 ratio (extract dissolved in $1 \%$ Tween $80: 1 \%$ Tween 80)(Table 2).

The amount of germinated spores at 1:0 ratio (extract dissolved in $1 \%$ Tween 80: 1\% Tween 80) was approximately twice the value presented in Table 1 for those extracts prepared from de $R$. graveolens, S. rebaudiana and T. vulgaris. Regarding $O$. manjorona, the difference between values shown in Table 1 and Table 2 was much smaller, but for Citrus sp. the percentage of germinated spores was about four times less in the former Table. Probably, it ocurred due to differences in the plant collecting period, which can influence metabolite production (SAITO et al., 2004).

To simplify the purification process to be employed in the future to isolate the active substances and to preserve them, it is desirable to eliminate humidity. One of the softest processes to do so is freeze-drying (PASTORINI et al., 2002), whose influence on plant extract activity was evaluated in this work. With a probability of 0.109 and a level of significance fixed in $5 \%$, it became clear after the Wilcoxon test that the freeze-drying process did not affect the antifungal activity (Table 3). 
Table 2 - Percentage of Colletotrichum gloeosporioides germinated spores after treatment with plant extracts at different concentrations.

\begin{tabular}{lccc}
\hline \multicolumn{3}{c}{ Germinated spores (\%) } \\
\hline Plant & $100 \%$ Extract & $50 \%$ Extract & $25 \%$ Extract \\
Citrus sp. & $23.6 \mathrm{c}$ & $98.3 \mathrm{c}$ & $97.6 \mathrm{a}$ \\
Ocimum gratissimum & $0.6 \mathrm{a}$ & $88.0 \mathrm{~b}$ & $97.0 \mathrm{a}$ \\
Origanum manjorona & $4.3 \mathrm{a}$ & $85.3 \mathrm{~b}$ & $94.0 \mathrm{a}$ \\
Ruta graveolens & $14.3 \mathrm{~b}$ & $85.3 \mathrm{~b}$ & $90.0 \mathrm{a}$ \\
Stevia rebaudiana & $42.3 \mathrm{~d}$ & $98.6 \mathrm{c}$ & $98.6 \mathrm{a}$ \\
Thymus vulgaris & $31.3 \mathrm{c}$ & $74.0 \mathrm{a}$ & $91.6 \mathrm{a}$ \\
$1 \%$ Tween 80 & $92.5 \mathrm{e}$ & $92.0 \mathrm{c}$ & $92.0 \mathrm{a}$ \\
\hline CV $(\%)=5.57$ & & &
\end{tabular}

Means of three replicates with the same letter in a column do not differ significantly $(P \leq 0.05)$

Table 3 - Percentage of Colletotrichum gloeosporioides germinated spores after treatment with plant extracts before and after freeze-drying.

\begin{tabular}{lccccc}
\hline \multicolumn{1}{c}{ Plant } & Part & \multicolumn{2}{c}{ Geminated spores $(\%)$} & & \multirow{2}{*}{ Posto de $d$} \\
\cline { 3 - 5 } & & Before & After & & \\
\hline Citrus limonia & Leaf & 0.0 & 2.0 & -2.00 & -9.5 \\
Citrus sp. & Leaf & 0.0 & 0.3 & -0.33 & -4.0 \\
Digitalis lanata & Flower & 0.0 & 0.6 & -0.66 & -6.0 \\
Digitalis lanata & Leaf & 0.0 & 1.0 & -1.00 & -7.0 \\
Ocimum gratissimum & Flower & 3.7 & 0.0 & 3.67 & 12.0 \\
Ocimum gratissimum & Leaf & 0.0 & 0.0 & 0.00 & 2.0 \\
Origanum manjorona & Leaf & 0.0 & 0.0 & 0.00 & 2.0 \\
Petiveria alliacea & Leaf & 0.0 & 2.7 & -2.67 & -11.0 \\
Ruta graveolens & Leaf & 0.0 & 1.3 & -1.33 & -8.0 \\
Stevia rebaudiana & Flower & 2.0 & 4.0 & -2.00 & -9.5 \\
Stevia rebaudiana & Leaf & 0.6 & 1.0 & -0.34 & -5.0 \\
Thymus vulgaris & Leaf & 0.0 & 0.0 & 0.00 & 2.0 \\
\hline T=18 & & & &
\end{tabular}

Statistical analysis was carried out with the Wilcoxon test for matched pairs.

\section{CONCLUSION}

Among the several extracts studied, those from $A$. eupatoria, Petiveria sp. and mainly from $D$. lanata, $P$. lanceolata and $S$. rebaudiana, afforded very promissing results to be used for the control of $C$. gloeosporioides. The most active extract was that from $O$. manjorona, which inhibited $96 \%$ of Colletotrichum gloeosporioides spore germination.

\section{ACKNOWLEDGEMENTS}

To Prof. Mário Sobral de Abreu (DFP-UFLA), Who kindly permitted the use of his Laboratory to carry out the experiments with the fungus. 


\section{REFERENCES}

ABOU-JAWDAH, Y.; SOBH, H.; SALAMEH, A. Antimycotic activities of selected plant flora, growing wild in lebanon, against phytopathogenic fungi. Journal of Agricultural and Food Chemistry, Washington, v. 50, n. 11, p. 3208-3213, May 2002.

ADAM, K.; ADAM, K.; SIVROPOULOU, A.; KOKKINI, S.; LANARAS, T.; ARSENAKIS, M. Antifungal activities of Origanum vulgare subsp. hirtum, Mentha spicata, Lavandula angustifolia, and Salvia fruticosa essential oils against human pathogenic fungi. Journal of Agricultural and Food Chemistry, Washington, v. 46, n. 5, p. 1739-1745, May 1998.

BAFI-YAEBOA, N. F. A.; ARNASON, J. T.; BAKER, J.; SMITH, M. L. Antifungal constituents of Northern prickly ash, Zanthoxylum americanum Mill. Phytomedicine, Jena, v. 12 , n. 5, p. 370-377, May 2005.

BANZATTO, D. A.; KRONKA, N. S. Experimentação agrícola. Jaboticabal: Funep, 1989. 245 p.

BENEVIDES, P. J. C.; YOUNG, M. C. M.; GIESBRECHT, A. M.; ROQUE, N. F.; BOLZANI, V. D. S. Antifungal polysulphides from Petiveria alliacea L. Phytochemistry, Oxford, v. 57, n. 5, p. 743-747, July 2001.

BRASIL. Ministério da Agricultura, Pecuária e Abastecimento. Agronegócio brasileiro: uma oportunidade de investimentos. Disponível em: <http:// www.agricultura.gov.br>. Acesso em: 8 set. 2005.

CACERES, A.; LOPEZ, B.; JUAREZ, X.; DELAGUILA, J.; GARCIA, S. Plants used in Guatemala for the treatment of dermatophytic infections: 2. evaluation of antifungal activity of 7 american plants. Journal of Ethnopharmacology, Clare, v. 40, n. 3, p. 207-213, Dec. 1993.

CARVALHO, G. A. Efeito in vitro e in vivo de filtrados de rizobactérias sobre Colletotrichum gloeosporioides Penz. do cafeeiro. 2004. 55 p. Dissertação (Mestrado em Agronomia) - Universidade Federal de Lavras, Lavras, 2004.

CORTEZ, D. A. G.; YOUNG, M. C. M.; MARSTON, A.; WOLFENDER, J. L.; HOSTETTMANN, K. Xanthones, triterpenes and a biphenyl from Kielmeyera coriacea. Phytochemistry, Oxford, v. 47, n. 7, p. 1367-1374, Apr. 1998.
DAMME, E. J. M. van; CHARELS, D.; MENUBOUAOUICHE, L.; PROOST, P.; BARRE, A.; ROUGE, P.; PEUMANS, W. J. Biochemical, molecular and structural analysis of multiple thaumatin-like proteins from the elderberry tree (Sambucus nigra L.). Planta, New York, v. 214, n. 6, p. 853-862, Apr. 2002.

FERREIRA, J. B. Flutuação sazonal e associações de Colletotrichum gloeosporioides Penz. a diferentes órgãos e tecidos de cafeeiros ( Coffea arabica L.). 2004. 90 p. Dissertação (Mestrado em Agronomia) - Universidade Federal de Lavras, Lavras, 2004.

FERREIRA, D. F. Análises estatísticas por meio do Sisvar para Windows versão 4.0. (Paper presented at the 45a Reunião AnuAL da Região Brasileira da Sociedade Internacional de Biometria, São Carlos. 2000.

FICKER, C. E.; SMITH, M. L.; SUSIARTI, S.; LEAMAN, D. J.; IRAWATI, C.; ARNASON, J. T. Inhibition of human pathogenic fungi by members of Zingiberaceae used by the Kenyah (Indonesian Borneo). Journal of Ethnopharmacology, Clare, v. 85, n. 2/3, p. 289-293, Apr. 2003.

FIORI, A. C. G.; SCHWAN-ESTRADA, K. R. F.; STANGARLIN, J. R.; VIDA, J. B.; SCAPIM, C. A.; CRUZ, M. E. S.; PASCHOLATI, S. F. Antifungal activity of leaf extracts and essential oils of some medicinal plants against Didymella bryoniae. Journal of PhytopathologyPhytopathologische Zeitschrift, Berlin, v. 148, n. 7/8, p. 483-487, Aug. 2000.

GIAMPERI, L.; FRATERNALE, D.; RICCI, D. The in vitro action of essential oils on different organisms. Journal of Essential Oil Research, Carol Stream, v. 14, n. 4, p. 312318, July/Aug. 2002.

HOLETZ, F. B.; PESSINI, G. L.; SANCHES, N. R.; CORTEZ, D. A. G.; NAKAMURA, C. V.; DIAS, B. P. Screening of some plants used in the brazilian folk medicine for the treatment of infectious diseases. Memórias do Instituto Oswaldo Cruz, Rio de Janeiro, v. 97, n. 7, p. 1027-1031, Oct. 2002.

KAWAI, K.; BEPPU, H.; SHIMPO, K.; CHIHARA, T.; YAMAMOTO, N.; NAGATSU, T.; UEDA, H.; YAMADA, $\underline{Y}$. In vivo effects of Aloe arborescens Miller var. natalensis Berger (Kidachi aloe) on experimental tinea pedis in guineapig feet. Phytotherapy Research, Sussex, v. 12, n. 3, p. 178182, May 1998. 
KISHORE, N.; CHANSOURIA, J. P. N.; DUBEY, N. K. Antidermatophytic action of the essential oil of Chenopodium ambrosioides and an ointment prepared from it. Phytotherapy Research, Sussex, v. 10, n. 5, p. 453455, Aug. 1996.

LIMA, E. D.; GOMPERTZ, O. F.; PAULO, M. D.; GIESBRECHT, A. M. In vitro antifungal activity of essential oils against clinical isolates of dermatophytes. Revista de Microbiologia, São Paulo, v. 23, n. 4, p. 235-238, Oct./Dec. 1992.

LU, Y. H.; ZHANG, Z.; SHI, G. X.; MENG, J. C.; TAN, R. X. A new antifungal flavonol glycoside from Hypericum perforatum. Acta Botanica Sinica, Beijing, v. 44, n. 6, p. 743-745, June 2002.

MIRANDA, E. F. O. Caracterização morfológica, molecular, bioquímica e patogênica de isolados de Colletotrichum spp. associados ao cafeeiro em Minas Gerais e comparação com Colletotrichum Kahawae. 2003. 147 p. Tese (Doutorado em Agronomia) - Universidade Federal de Lavras, Lavras, 2003.

MISHRA, A.; DUBEY, N. K.; SINGH, S.; CHATURVEDI, C. M. Biological activities of essential oil of Chenopodium Ambrosioides against storage pests and its effect on puberty attainment in Japanese quail. National Academy Science Letters-India, Allahabad, v. 25, n. 5/6, p. 176-179, 2002.

NINO, J.; ESPINAL, C. M.; MOSQUERA, O. M.; CORREA, Y. M. Antimycotic activity of 20 plants from Colombian flora. Pharmaceutical Biology, Lisse, v. 41, n. 7, p. 491-496, Oct. 2003.

OLIVA, A.; MEEPAGALA, K. M.; WEDGE, D. E.; HARRIES, D.; HALE, A. L.; ALIOTTA, G.; DUKE, S. O. Natural fungicides from Ruta graveolens L. leaves, including a new quinolone alkaloid. Journal of Agricultural and Food Chemistry, Washington, v. 51, n. 4, p. 890-896, Feb. 2003.

PASTORINI, L. H.; BACARIN, M. A.; ABREU, C. M. Secagem de material vegetal em forno de microondas para determinação de matéria seca e análises químicas. Ciência e Agrotecnologia, Lavras, v. 26, n. 6, p. 1252-1258, Nov./ Dec. 2002.

PATRA, M.; SHAHI, S. K.; MIDGELY, G.; DIKSHIT, A. Utilization of essential oil as natural antifungal against nail- infective fungi. Flavour and Fragrance Journal, Sussex, v. 17, n. 2, p. 91-94, Mar./Apr. 2002.

PESSINI, G. L.; DIAS, B. P.; NAKAMURA, C. V.; CORTEZ, D. A. G. Antifungal activity of the extracts and neolignans from Piper regnellii (Miq.) C. DC. var. pallescens (C. DC.) Yunck. Journal of the Brazilian Chemical Society, São Paulo, v. 16, n. 6A, p. 1130-1133, Nov./Dec. 2005.

POPOVIC, M.; JAKOVLJEVIC, V.; BURSAC, M.; MITIC, R.; RASKOVIC, A.; KAURINOVIC, B. Biochemical investigation of yarrow extracts (Achillea millefolium L.). Oxidation Communications, Sofia, v. 25, n. 3, p. 469-475, 2002.

ROTH, G. N.; CHANDRA, A.; NAIR, M. G. Novel bioactivities of Curcuma longa constituents. Journal of Natural Products, Washington, v. 61, n. 4, p. 542-545, Apr. 1998.

SAITO, M. L.; POTT, A.; FERRAZ, J. M. G.; NASCIMENTO, R. dos S. Avaliação de plantas com atividade deterrente alimentar em Spodoptera frugiperda (J.E.Smith) e Anticarsia gemmatalis Hubner. Pesticidas: Revista de Ecotoxicologia e Meio Ambiente, Curitiba, v. 14, p. 1-10, 2004

SCOTT, A. J.; KNOTT, M. A cluster analysis method for grouping means in the analysis of variance. Biometrics, Washington, v. 30, n. 3, p. 507-512, Sept. 1974.

SENER, B. Recent results in the search for bioactive compounds from Turkish medicinal-plants. Pure and Applied Chemistry, Oxford, v. 66, n. 10/11, p. 2295-2298, Oct./Nov. 1994.

SHIN, S. Anti-Aspergillus activities of plant essential oils and their combination effects with ketoconazole or amphotericin B. Archives of Pharmacal Research, Seoul, v. 26, n. 5, p. 389-393, May 2003.

SHRIVASTAVA, J. N.; KUSHWAHA, R. K. S.; SRIVASTAVA, J. N.; SHUKLA, J. P. Antifungal activity of Parthenium-Hysterophorus Linn. Current Science, Bangalore, v. 53, n. 13, p. 712-712, 1984.

SHUKLA, Y. N.; SRIVASTAVA, A.; KUMAR, S. Aryl lipid and triterpenoid constituents from Oenothera biennis. Indian Journal of Chemistry Section B-Organic Chemistry Including Medicinal Chemistry, New Delhi, v. 38, n. 6, p. 705-708, June 1999. 
SIEGEL, S. Estatística não-paramétrica para ciências do comportamento. São Paulo: McGraw-Hill, 1977. 350 p.

SOUZA, E. L. de; LIMA, E. D.; FREIRE, K. R. D.; SOUSA C. P. de. Inhibitory action of some essential oils and phytochemicals on the growth of various moulds isolated from foods. Brazilian Archives of Biology and Technology, Curitiba, v. 48, n. 2, p. 245-250, 2005.

THANGAVELU, R.; SUNDARARAJU, P.; SATHIAMOORTHY, S. Management of anthracnose disease of banana caused by Colletotrichum musae using plant extracts. Journal of Horticultural Science \& Biotechnology, Kent, v. 79, n. 4, p. 664-668, July 2004.

TIWARI, T. N.; CHANSOURIA, J. P. N.; DUBEY, N. K. Antimycotic potency of some essential oils in the treatment of induced dermatomycosis of an experimental animal. Pharmaceutical Biology, Lisse, v. 41, n. 5, p. 351356, Aug. 2003. 